\title{
A quantitative study of magnetospheric magnetic field line deformation by a two-loop substorm current wedge
}

\author{
A. V. Nikolaev ${ }^{1}$, V. A. Sergeev ${ }^{1}$, N. A. Tsyganenko ${ }^{1}$, M. V. Kubyshkina ${ }^{1}$, H. Opgenoorth ${ }^{2}$, H. Singer ${ }^{3}$, and \\ V. Angelopoulos 4 \\ ${ }^{1}$ Department of Earth Physics, Saint Petersburg State University, Petrodvoretz, Russia \\ ${ }^{2}$ Uppsala Division, Swedish Institute of Space Physics, Uppsala, Sweden \\ ${ }^{3}$ Space Weather Prediction Center, NOAA, Boulder, Colorado, USA \\ ${ }^{4}$ Department of Earth, Planetary, and Space Sciences and Institute of Geophysics and Space Physics, \\ University of California, Los Angeles, California, USA
}

Correspondence to: A. V. Nikolaev (demosfen.spb@gmail.com)

Received: 24 September 2014 - Revised: 8 March 2015 - Accepted: 2 April 2015 - Published: 29 April 2015

\begin{abstract}
Substorm current wedge (SCW) formation is associated with global magnetic field reconfiguration during substorm expansion. We combine a two-loop model SCW (SCW2L) with a background magnetic field model to investigate distortion of the ionospheric footpoint pattern in response to changes of different SCW2L parameters. The SCW-related plasma sheet footprint shift results in formation of a pattern resembling an auroral bulge, the poleward expansion of which is controlled primarily by the total current in the region 1 sense current loop $\left(I_{1}\right)$. The magnitude of the footprint latitudinal shift may reach $\sim 10^{\circ}$ corrected geomagnetic latitude (CGLat) during strong substorms $\left(I_{1}=2 \mathrm{MA}\right)$. A strong helical magnetic field around the field-aligned current generates a surge-like region with embedded spiral structures, associated with a westward traveling surge (WTS) at the western end of the SCW. The helical field may also contribute to rotation of the ionospheric projection of narrow plasma streams (auroral streamers). Other parameters, including the total current in the second (region 2 sense) loop, were found to be of secondary importance. Analyzing two consecutive dipolarizations on 17 March 2010, we used magnetic variation data obtained from a dense midlatitude ground network and several magnetospheric spacecraft, as well as the adaptive AM03 model, to specify SCW2L parameters, which allowed us to predict the magnitude of poleward auroral expansion. Auroral observations made during the two substorm activations demonstrate that the SCW2L combined with the AM03 model nicely describes the azimuthal progression and the observed magnitude of the au-
\end{abstract}

roral expansion. This finding indicates that the SCW-related distortions are responsible for much of the observed global development of bright auroras.

Keywords. Magnetospheric physics (auroral phenomena; current systems; storms and substorms)

\section{Introduction}

Electric currents flowing along the magnetic field lines (fieldaligned currents, FACs), known since K. Birkeland's studies in the early 1900s, are an important part of the threedimensional current system that is responsible for the configuration of the dynamical magnetosphere. The most stable well-known component of this current system, the largescale double-layer system of region 1 (R1) and region 2 (R2) FACs (Iijima and Potemra, 1976), supports magnetospheric convection and the twin-vortex pattern of the equivalent ionospheric current. Another well-known current system, the substorm current wedge (SCW), is responsible for major magnetotail reconfigurations during magnetospheric substorms. The SCW concept was first introduced by McPherron et al. (1973), who described an SCW as a 3-D current system, connecting the disrupted tail current with the ionosphere via the downward/upward R1 type FACs (at the dawn/dusk sides, respectively). Their single-loop SCW model not only qualitatively explained observed magnetotail field dipolarizations, but also provided realistic magnitudes and polarity of bay-like magnetic field variations at midlatitudes. There- 
fore, Horning et al. (1974), Sergeev et al. (1996), and Chu et al. (2014) have suggested that it be used as a tool to monitor the intensity and location of substorm currents. Sergeev et al. (2011) proposed a corresponding single-wedge SCW model with a realistic shape of the current-carrying magnetic field lines and filamentary (rather than linear) currents suitable for magnetospheric studies.

Theoretical and observational studies however, now require that the single-wedge SCW model be updated by including an additional element, a loop with oppositely directed current (in the R2 sense) spanning nearly the same longitudinal sector as the primary R1 current loop, but located closer to Earth. The single-wedge model thus becomes a twoloop (quadrupolar FAC) SCW model (SCW2L) (Sergeev et al., 2014a). Magnetohydrodynamic simulations of magnetotail reconnection (Birn and Hesse, 1999; Birn and Hesse, 2014), the recent equilibrium Rice Convection Model (RCME), and flow burst simulations (Yang et al., 2012) consistently show that a plasma pressure increase in front of the earthward flow channel is responsible for generation of this additional R2 loop. From observations, Liu et al. (2013) inferred quadrupolar FACs by analyzing individual propagating flow bursts, while Sergeev et al. (2011) and Sergeev et al. (2014a) showed them to be consistent with SCW-related magnetic perturbations by comparing multipoint observations in the near magnetosphere and at ground midlatitudes during substorms.

During strong substorms, the total SCW current may exceed $1 \mathrm{MA}$ and thus may significantly change magnetospheric configuration and ionospheric mapping in the nearEarth region. At the same time, intense FAC sheets (especially upward FACs) accelerate precipitating electrons that cause bright auroras (Waters et al., 2001). Therefore, substorm-related magnetic reconfiguration and associated changes in field-line mapping from the magnetosphere to the ionosphere should be reflected in an auroral pattern redistribution, which, in turn, can provide information about these changes. An example is poleward auroral expansion accompanied by formation of a bulge of bright auroras. This basic signature of the substorm expansion phase has been traditionally related to electric current disruption and magnetic reconnection in the magnetotail (e.g., Roux et al., 1991; Yahnin et al., 2006; Kubyshkina et al. 2011). The relationship is based on rather limited evidence however (see, e.g., summary by Keiling et al., 2012). The quantitative evaluation of the role of FACs in variable patterns of field-line mapping during substorm-related dipolarization and auroral expansion, an interesting exercise, has been addressed in a few previous papers. Those studies, however, utilized highly idealized SCW models with infinitely thin line currents flowing along either dipolar or early empirical model field lines (Vasilyev et al., 1986; Kaufmann and Larson, 1989; Tsyganenko, 1997), or used dipolarization effects to represent a global tail reconfiguration (Kubyshkina et al., 2011). Because SCW effects on the magnetotail configuration and mapping by applying the upgraded (quadrupolar) SCW2L model is an promising approach, we do so in this paper.

In Sect. 2, we quantitatively investigate SCW-related deformations, emphasizing mapping from the equatorial magnetosphere to the ionosphere; determine the impact of adding the SCW2L model on the deformation magnitude; and discuss expected manifestations in the auroral observations. In Sect. 3, we evaluate the expected poleward expansion based on time-varying, data-adaptive magnetospheric models (using the SCW2L and the AM03 adaptive model) and compare their results with observed magnitude of poleward expansion for the isolated substorm event, taking the advantage of excellent spacecraft coverage in the magnetosphere.

\section{Modeling of the ionospheric footprint displacement produced by the substorm current wedge}

\subsection{Brief description of the SCW2L model}

Here we use the SCW2L model, which was presented, tested, and extensively discussed by Sergeev et al. (2014a) (hereafter referred to as Paper 1). It includes two pairs of fieldaligned currents: the high-latitude R1 loop and the more earthward/equatorward R2 loop (see Fig. 1a). When combined, these loops form a quadrupolar FAC source near the ionosphere. The model was developed for substorm case studies to quantitatively evaluate the intensities of both R1 $\left(I_{1}\right)$ and R2 $\left(I_{2}\right)$ currents, based on observations. Its input includes the dipolarization amplitude $\left(\Delta B_{Z}\right)$ in the magnetosphere, measured by a few spacecraft, and the amplitudes of $\Delta X$ and $\Delta Y$ components of bay-like variations, measured at several ground midlatitude observatories. As discussed in Paper 1 and illustrated in Fig. 1c, the magnitude $\Delta B_{Z}$ in the magnetosphere is rather uniform over most of the dipolarized region, that is, in the area between the $\mathrm{R} 1$ and $\mathrm{R} 2$ loops (red hatching in Fig. 1d). Here $\Delta B_{Z}$ is mostly sensitive to the magnitude of R1 current $\left(I_{1}\right)$ and allows one to evaluate its magnitude, whereas the midlatitude ground variations respond to the net current $\left(I_{1}+I_{2}\right)$, so the combination of two (magnetospheric and ground-based) inputs is necessary to evaluate both $I_{1}$ and $I_{2}$ parameters.

Because of the scarcity of magnetospheric spacecraft observations, the model should be as simple as possible. In particular, both R1 and R2 loops are assumed to occupy the same azimuthal sector, which is roughly consistent with simulation results presented by Yang et al. (2012) and Birn and Hesse (2013). In our case, we use the filamentary current model with the current transverse spread specified as $D_{0} \sim R^{3 / 2}$, which amounts to 1 to $2 \mathrm{Re}$ in the region of interest, with the FACs flowing along the realistic magnetic field lines of the T89 model (Tsyganenko et al., 1989). The stretch of the background magnetic field (used to trace the FACs) is controlled by the RCF parameter, which varies between 0 (for a quiet dipolar-like configuration) and 8 (for a very stretched tail-like configuration) in the T89 code. 

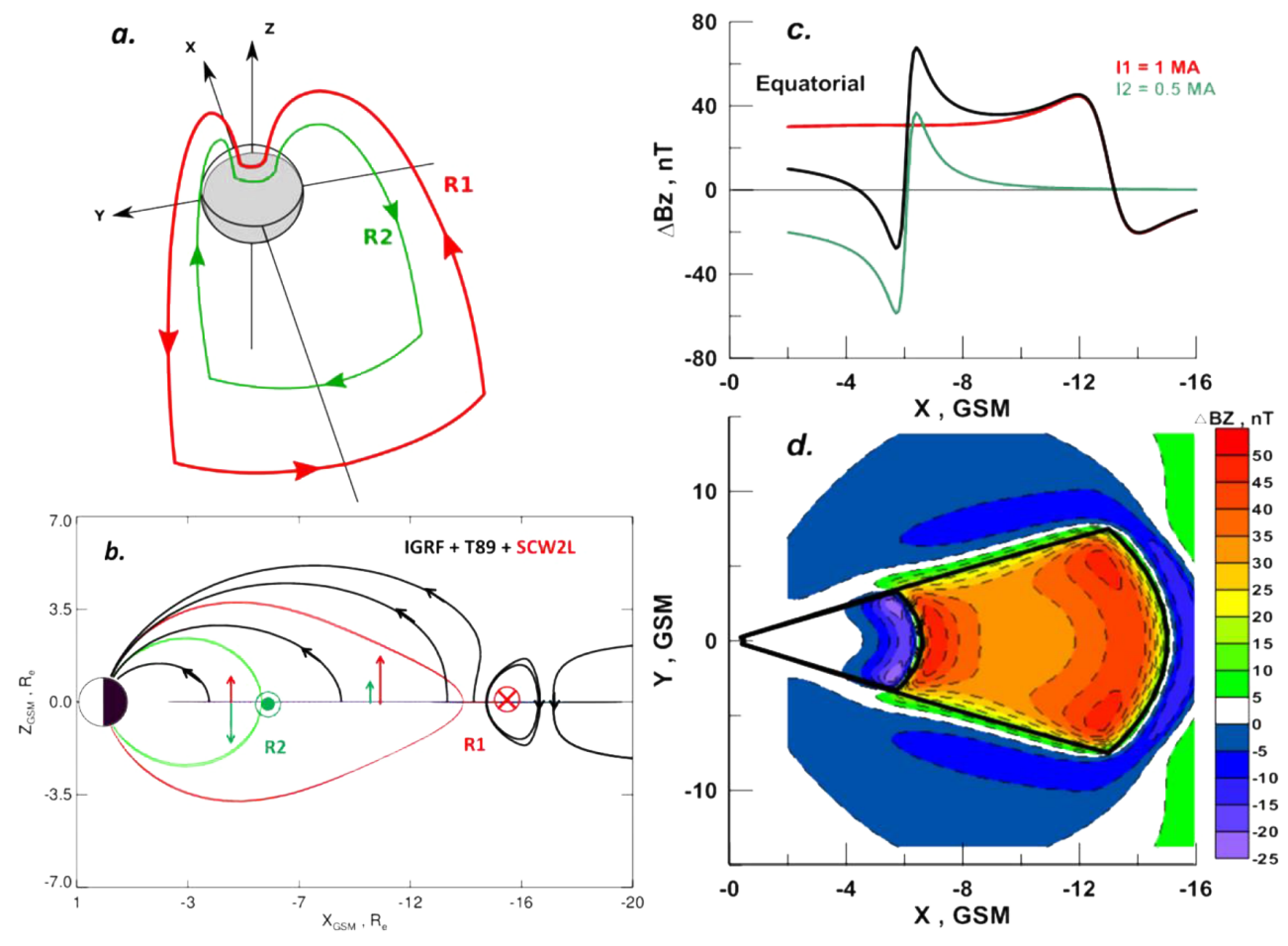

Figure 1. (a) Illustration of the two-loop SCW model (SCW2L); (b) magnetic field-line topology when the background (T89+ IGRF) magnetic field (shown by current-carrying red/green lines) is compared to field lines (black lines) after the addition of SCW2L; (c) partial contribution to $\Delta B_{Z}$ perturbations of R1 (red) and R2 (green) SCW components and their sum (black line); (d) $\Delta B_{Z}$ distribution in the equatorial plane in the SCW2L model (adapted from Sergeev et al., 2014a). All illustrations are done for a $60^{\circ}$ wide wedge with $I_{1}=1 \mathrm{MA}$ and $I_{2}=0.5 \mathrm{MA}$.

With $\mathrm{RCF}=0$, an equatorial point at $X_{\mathrm{GSM}}=-15 \mathrm{RE}$, $Y_{\mathrm{GSM}}=0$ in the neutral sheet maps to geomagnetic latitude of $\sim 70^{\circ}$ GLat in the ionosphere, while setting $\mathrm{RCF}=6$ moves the footprint down to $\sim 63^{\circ}$ GLat (see also Sergeev et al. (2011) for the detailed description of RCF parameter). Using the filamentary model rather than the azimuthally distributed field-aligned currents does not significantly affect $\Delta B_{Z}$ in the dipolarized region, except in the immediate vicinity of the FAC filament (see Fig. 3 and supplementary plots S1-S3 in Sergeev et al., 2014a). Also, in our analyses below we set the distance to the equatorial current of the $\mathrm{R} 1$ loop to $\mathrm{RT}_{1}=15 \mathrm{Re}$ (see also parameter descriptions in Fig. S1 in the Supplement).

Figure $1 \mathrm{~b}$ illustrates the applicability domain of the SCW2L model, limited primarily to the inner magnetosphere, where the model is intended to faithfully describe the dipolarization effects. Close to the filamentary equatorial R1 current and beyond it, the model cannot provide a realistic description of electric currents flowing in the reconnecting plasma sheet. Nevertheless, as shown in Paper 1, the amplitude and distribution of the dipolarization amplitude $\Delta B_{Z}$ in the inner region (earthward of $12 \mathrm{Re}$ ) depends only slightly on the exact location and radial distribution of the equatorial $\mathrm{R} 1$ current. If the peak perturbation from the equatorial filament exceeds the background $B_{Z}$ value, a closed field line loop is formed around the filament (Fig. 1b). In addition, a region with southward total $B_{Z}$ that is topologically disconnected from the ionosphere is formed tailward of the R1 filament. These two model artifacts should be kept in mind when analyzing SCW2L-related deformation and magnetic field-lines mapping.

\subsection{SCW2L-related deformations}

Both the background magnetic field (IGRF + T89) and SCW2L perturbations are three-dimensional vector fields with complex distribution and geometry. To quantitatively characterize magnetic field deformations associated with SCW onset, we use a method in which a regular set of contours in the nightside equatorial plane is mapped along field lines onto the ionosphere. We then evaluate changes in the mapping patterns caused by the adding of the SCW model contribution to the background field. More specifically, the reference contours are chosen as a family of equatorial arcs, i.e., geocentric circular segments in the $Z=0$ plane (with the geodipole tilt angle assumed to be zero), distributed between 4 and $20 \mathrm{Re}$, as shown in Fig. 2. Each point on the arc is mapped to the ionosphere $(R=1.02 \mathrm{Re})$ twice: first, using only the background IGRF + T89 field, and then using the IGRF + T89 + SCW2L model with the substorm wedge 


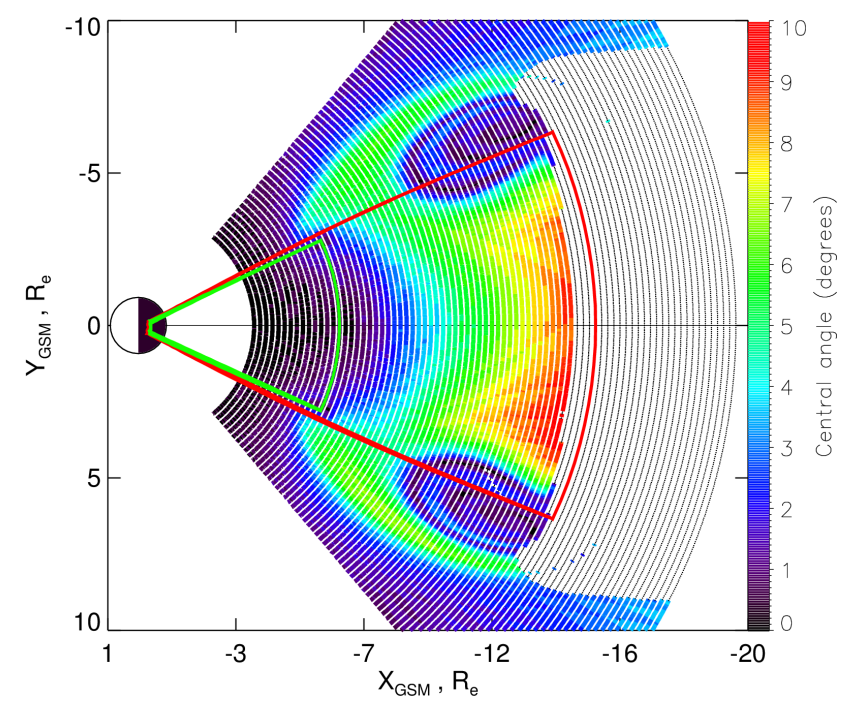

Figure 2. Magnetospheric pattern of neutral sheet footprint geocentric angular displacement (color coded) caused by addition of the SCW2L model to the T89+ IGRF models. The SCW2L model parameters were set as follows: $I_{1}=1 \mathrm{MA}, I_{2}=0.5 \mathrm{MA}$, $\mathrm{RT}_{1}=15 \mathrm{Re}, \mathrm{RT}_{2}=6 \mathrm{Re}, \mathrm{RCF}=6$, and the wedge azimuthal width $50^{\circ}$.

contribution added. The azimuthal and longitudinal shift between the footpoints

$\alpha=\cos ^{-1}\left(\frac{x_{1} x_{2}+y_{1} y_{2}+z_{1} z_{2}}{R^{2}}\right)$,

serves as a quantitative measure of the SCW mapping effect. Here $R=1.02 \mathrm{Re}$, and $\left[x_{1}, y_{1}, z_{1}\right]$ and $\left[x_{2}, y_{2}, z_{2}\right]$ are footprint Cartesian coordinates, obtained from the two tracings. An example of the footprint shift distribution in the equatorial plane is shown in Fig. 2; each point on the arc is colored according to its $\alpha$ value. As mentioned above, the valid area in our analysis is the region earthward of the R1 equatorial current between the upward and downward FACs and colored in green and red lines. No ionospheric footpoints exist tailward of that area (grey color) because the corresponding field lines either belong to the magnetic "island" inside the loop or map tailward.

The equatorial diagram of the footpoint shifts should be viewed with the ionospheric mapping pattern shown in Fig. 3 aimed to characterize its geometry. At the ionospheric level, we show two families of reference equatorial equidistant arcs mapped to the ionosphere using only the background model field (red contours) as well as the background and the SCW2L field (green curves). Such a representation, which helps to show different types of magnetic field deformation; it is especially useful when discussing possible auroral implications.

Comparing Figs. 2 and 3 reveals three specific regions of footprint deformation. The first, the most important region inside the dipolarized region, lies between the equatorial R1

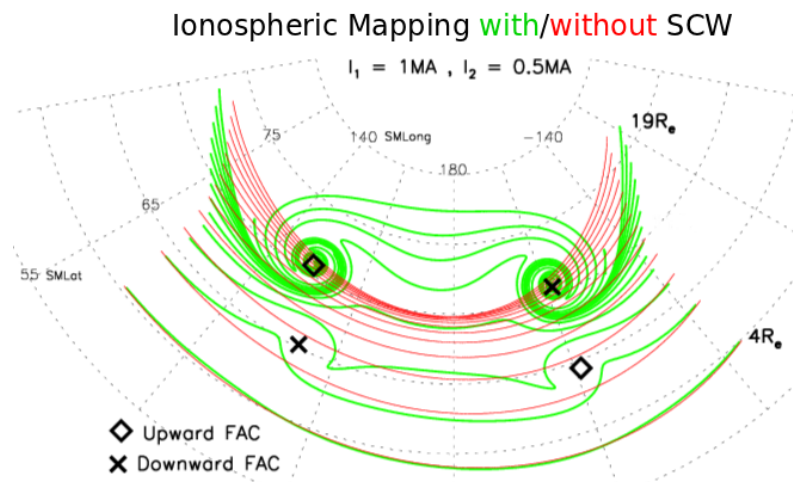

Figure 3. Ionospheric mapping of equatorial geocentric circle arcs (equatorial distance is labeled for selected circles). The red lines show neutral sheet mapping using the T89 + IGRF model; the green lines show mapping of the same points using the SCW2L + T89 + IGRF model. Ionospheric locations of upward (downward) FACs are indicated by diamond (cross) symbols. The SCW2L model is the same as in Fig. 2.

and R2 currents. Here the SCW2L-related $\Delta B_{Z}$ perturbations are significantly larger than the background $B_{Z}$ component $\left(B_{Z 0}\right)$ : the traced field lines pass at larger distances from the neutral sheet plane (see Fig. 1b) and hence land at higher latitudes. At the ionospheric level, the dipolarization region corresponds to the area of significant poleward shift of the footpoints. In the example illustrated in Figs. 2 and 3, this shift can reach up to $8^{\circ}$ corrected geomagnetic latitude (CGLat) at the center of the SCW. Because of an increase in the $\Delta B_{Z} / B_{Z 0}$ ratio, the magnitude of the poleward expansion increases as the equatorial point approaches the R1 filament.

The second region corresponds to the field-line twisting area around the intense R1 field-aligned current filament, which is well represented by the spiral-like shapes in Fig. 3. Initially located within the wedge close to the filament axis, the points are significantly twisted, but their resulting footpoint displacements appear to be small, forming the blue areas near the FACs (between 8 and 14 Re along the $x$ axis) in Fig. 2. Contradictions between the amount of footprint movement and small $\alpha$ values appear in the cases when footpoints rotate around FACs and return close to the original location. The combination of type 1 and type 2 deformations produces a large-scale poleward bulge-like structure in the ionospheric projection of the magnetospheric dipolarization region, which may be associated with the auroral bulge.

The third deformation region is co-located with the footprint equatorward shift near the R2 equatorial current (here we placed it at the distance $\mathrm{RT}_{2}=6 \mathrm{Re}$ ). The effect of the footpoint twisting around the corresponding FACs results in an equatorward bulge-like ionospheric pattern. This bulge is several times smaller than the poleward bulge for two reasons: (1) a much stronger background field in the inner mag- 
netosphere, where the R2 loop is formed and (2) an R2 current that is smaller than the $\mathrm{R} 1$ current.

For auroral research, it is also instructive to map another type of neutral sheet contour. Rather than arc-like segments, one may consider rectilinear strips oriented along the $x$ axis plasma flow geometry (Fig. 4a) and created as azimuthally localized partitions of the neutral sheet described in Sect. 2.2. The distorted ionospheric projections of these strips can be likened to elementary structures associated with auroral arcs or other features observed at low altitudes. One such structure can be an ionospheric projection of narrow (2-3 Re wide across the tail), fast plasma streams, also known as "bursty bulk flows" (BBFs) (Baumjohann et al., 1990; Angelopoulos et al., 1992), which are associated with a family of approximately north-south aligned auroral arcs (or auroral streamers, or poleward boundary intensifications, PBIs) (e.g., Elphinstone et al., 1996; Henderson et al., 1998; Lyons et al., 1999; Nakamura et al., 2001; Henderson, 2012, and references therein). Figure $4 \mathrm{a}$ shows an equatorial view of three line segments (I and II contours) in the dipolarized region, which simulate three hypothetical fast earthward plasma streams. Although their mapped images look similar, if mapped along the background magnetic field (Fig. 4b, black contours), adding the SCW2L contribution causes a significant deformation of the image. Although it is shifted poleward and somewhat elongated, the shape of the stream located at the central wedge meridian $(Y=0$, contour I) does not change much. The shape of the off-center contours (II), however, changes considerably, including a significant rotation of the mapped structure. Although the general features of the deformation are recognizable with the help of Fig. 3, the amount of rotation and the scale of the crescent-like structure depend on many details of structure location relative to the wedge field-aligned currents. In particular, the footpoints of the dawnside stream that crosses the equatorial projection of the wedge (but does not intersect the FAC flux tube) are subject to stronger rotation than those of a non-crossing duskside stream. In addition, the latitude of the stream endpoint (the most earthward) is roughly $4^{\circ}$ CGLat southward of the noncrossing stream's endpoint. Investigation of corresponding auroral patterns may have interesting implications for studies of the FAC strength and distribution.

\subsection{Poleward footpoint expansion as a function of SCW parameters}

In this section, we investigate poleward shifts of ionospheric footpoints traced from the neutral sheet at the central wedge meridian (here $Y=0, Z=0$ ) using different SCW2L parameter values. Values of the SCW2L spatial parameters, such as $P_{W}, P_{E}, \mathrm{RT}_{1}$, and $\mathrm{RT}_{2}$, are similar to those used in the previous section. Characterizing the event strength by a combination of R1 current intensity $\left(I_{1}\right)$ and field-line stretching amplitude (RCF), we select combinations corresponding to different magnetic disturbance levels as follows: weak substorms $I_{1}=0.5 \mathrm{MA}, \mathrm{RCF}=3$ (Fig. $5 \mathrm{c}$ ); moderate substorms $I_{1}=1 \mathrm{MA}, \mathrm{RCF}=6$ (Fig. $5 \mathrm{~d}$ ); and strong substorms $I_{1}=2 \mathrm{MA}, \mathrm{RCF}=8$ (Fig. 5e). To set the RCF dependence on $I_{1}$, we relied on Fig. 10 in Sergeev et al. (2014a), which demonstrated a statistical relationship between dipolarization amplitudes and $B_{Z 0}$ at geosynchronous distance prior to the dipolarization onset and suggested $I_{2} / I_{1}=0.5$.

As seen from Fig. 5c, d, and e, the parameter that effectively controls magnitude of poleward expansion is the intensity of the R1 current. The computed maximal poleward shift of ionospheric footpoints at the wedge central meridian is rather small in the case of weak substorm ( $\Delta$ Lat $\sim 2$ $3^{\circ}$ CGLat). It increases under moderate substorm conditions ( $\Delta$ Lat $\sim 5-6^{\circ}$ CGLat) and can reach $\Delta$ Lat $\sim 10^{\circ}$ CGLat during highly disturbed events. Such values look quite realistic when compared to the known magnitudes of the auroral poleward expansion during substorms (Akasofu, 1976).

Surprisingly, the growth of the R2 current (increase in the $I_{2} / I_{1}$ ratio under a fixed value of $I_{1}$ ), which enhances the dipolarization in the equatorial plane (see Fig. 1c, d, and e), actually decreases footprint shifts, resulting in a $20 \%$ smaller latitudinal expansion. This is explained by Fig. $5 b$, which shows the wedge-related $\Delta B_{Z}$ at locations along the magnetic field line, corresponding to the T89 + IGRF model and starting in the middle of the wedge from $X=-10 \mathrm{Re}$, $Z=0$. Figure $5 \mathrm{~b}$ shows that the increase in $I_{2}$ actually suppresses $\Delta B_{Z}$ in the high-latitude part of the field line (at small radial distances, $R<6 \mathrm{Re}$ ), without a substantial increase in $\Delta B_{Z}$ at its near-equatorial $(R>6 \mathrm{Re})$ part. The configuration of such a distorted field line is illustrated in Fig. 5a. According to Fig. 5c, d, and e, the equatorial bulge caused by the R2 current is virtually absent when $I_{2} / I_{1}$ is small $(=0.3)$ and has a relatively small magnitude $\left(<\sim 1^{\circ} \Delta\right.$ Lat $)$ when $I_{2} / I_{1}=0.8$.

As shown in Fig. 5d, the role of background field line stretching is also very modest. The stretch increase from $\mathrm{RCF}=3$ to 6 reduces the ionospheric shift in the region of strong magnetic gradients (near the R1 and R2 type currents) by $\Delta$ Lat $\sim 1-2^{\circ}$ CGLat. This can be partly due to changing magnitudes of the background $B_{Z 0}$ at different RCF, which resulted in an equatorward shift of the field-line footpoints.

\section{SCW and poleward auroral expansion during the 17 March 2010 substorm}

Realistic modeling of magnetospheric field deformation is an important step to validate the SCW2L model itself and quantitatively testing its prediction of the poleward expansion during substorms. Here we conduct such testing for the isolated substorm event on 17 March 2010, with excellent coverage of the magnetosphere by four Geostationary Operational Satellites (GOES), which monitored the nightside part of the synchronous orbit, and by four Time History of Events and Macroscale Interaction during Substorms spacecraft (Angelopoulos, 2008) in the tail. The event itself 

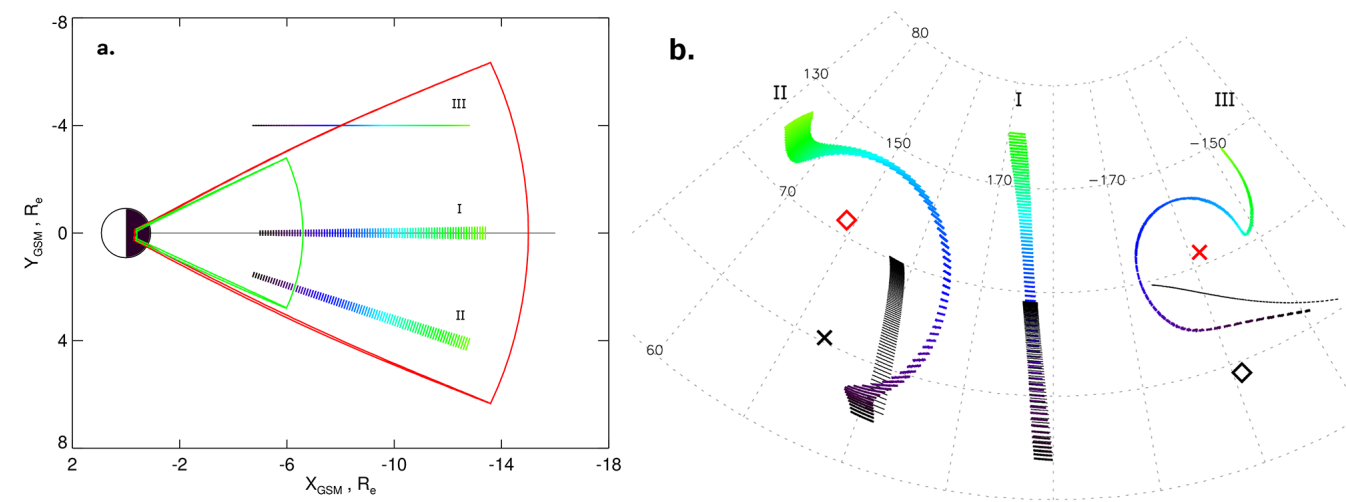

Figure 4. (a) Equatorial locations of three hypothetical narrow plasma streams; (b) their ionospheric footprints. The black strips indicate stream mapping using the background IGRF + T89 model; the colored strips represent stream mapping in the IGRF + T89 + SCW2L model $\left(I_{1}=1 \mathrm{MA}, I_{2}=0.5 \mathrm{MA}, \mathrm{R} 1=15 \mathrm{Re}, \mathrm{RT}_{2}=6 \mathrm{Re}\right.$ and $\left.\mathrm{RCF}=6\right)$. Color coding indicates the streamers, spatial orientation or movement direction.

and its spacecraft-based modeling (including SCW2L runs) are described in detail in a companion paper by Sergeev et al. (2014b). Here we briefly restate some of their results and concentrate on the mapping issues.

The inversion modeling was performed in two stages. In the first, we used the well-known magnetogram inversion algorithm (Sergeev et al., 1996) with a simple (based on dipolar field lines) SCW model and with input from 20 midlatitude magnetic observations, to infer the parameters of the SCW, symmetric (DR), and partial (DRP) ring current systems (the latter two systems changed little during that event, and their effect is not discussed here). In the second, we used values of westward (PW) and eastward (PE) SCW longitudes, obtained in the previous step, and ran the inversion procedure based on a combination of midlatitude ground-based data, spacecraft observations, and the advanced SCW2L model (see also Fig. S1 in the Supplement). The inversion algorithm usually searched for and found a global minimum of a fit function $\sigma$ :

$$
\begin{aligned}
\sigma=K_{\mathrm{ST}} \sum & \left(\left(\frac{\Delta X_{\mathrm{OBS}}}{K_{\mathrm{IND}}}-\Delta X_{\mathrm{MOD}}\right)^{2}\right. \\
& \left.+\left(\frac{\Delta Y_{\mathrm{OBS}}}{K_{\mathrm{IND}}}-\Delta Y_{\mathrm{MOD}}\right)^{2}\right) \\
& +K_{\mathrm{SC}} \sum\left(\Delta B_{\text {Zobs }}-\Delta B_{\text {Zmod }}\right)^{2},
\end{aligned}
$$

where the indices "obs" and "mod" stand for the observed and modeled fields, respectively, and $K_{\mathrm{IND}}=1.5$ is the induction correction coefficient. The summation is carried out over the $N_{\mathrm{ST}}$ stations and $N_{\mathrm{SC}}$ spacecraft, and $K_{\mathrm{ST}}$ and $K_{\mathrm{SC}}$ are the weight coefficients needed to balance the contributions to the minimized target function (i.e., $\left.K_{\mathrm{ST}} \times N_{\mathrm{ST}}=K_{\mathrm{SC}} \times N_{\mathrm{SC}}\right)$ from a large number of stations $\left(N_{\mathrm{ST}}=19\right)$ and a small number of spacecraft located inside the dipolarized region $\left(N_{\mathrm{SC}}=1\right.$ or 2$)$. Throughout that run, we kept some parameters fixed, including $\mathrm{RT}_{1}=15 \mathrm{Re}$,
$\mathrm{RT}_{\mathrm{DRP}}=13 \mathrm{Re}$, and $R_{\mathrm{DR}}=4 \mathrm{Re}$. We made equatorial distance to $\mathrm{R} 2$ current free and varied $\mathrm{RT}_{2}$ parameter between 5.5 and 6 Re.

As a result, we evaluated the $I_{1}, I_{2}$, and $I_{3}$ (DRP) currents for two consecutive dipolarizations with the activity starting at $T=04: 56 \mathrm{UT}$ (reference level, start time of activation no. 1) and $T=05: 36 \mathrm{UT}$ (start time of activation no. 2). The reference level for both activations was chosen at $\mathrm{T}_{0}=04: 56 \mathrm{UT}$ because the second activation started during the recovery from the first activation. The observed and modeled field perturbations during the peaks of two activations are compared in Fig. 6. This figure demonstrates good agreement between the observed and predicted dipolarization magnitudes, namely $\Delta B_{Z}$ in the bottom right panels for spacecraft dipolarization and $\Delta X$ and $\Delta Y$ components in the left panels for ground stations. We also ran the adaptive model AM03 (see also Sergeev et al. (2014b) for more details), which uses the T96 model equations but adjusts their parameters to provide a best fit to the magnetic field observed during the event of interest.

Using the inversion results, we can now predict the mapping using realistic parameters of the SCW2L model current system as we did in Sect. 2.2 (Figs. 7 and 8). The AM03 + IGRF model at 04:56 UT is used here as the background field model. Colored patterns in Fig. 7 illustrate the degree of footprint distortion (similarly to Fig. 2) for two dipolarization maxima epochs at 05:13 UT (left panel, event no. 1) and 05:50 UT (right panel, event no. 2).

Figure $8 \mathrm{~b}$ (colored lines) complements the previous figure by showing the time-varying latitude locations of GOES12 and 14 and THEMIS-A spacecraft footprints predicted by the SCW2L-based model. These footprints are compared to those predicted by the time-varying AM03 model (Fig. 8c, thin lines). To monitor the longitudinal location of these spacecraft relative to the SCW location, they are plotted in Fig. 8a. The mapping from a fixed neutral sheet location 


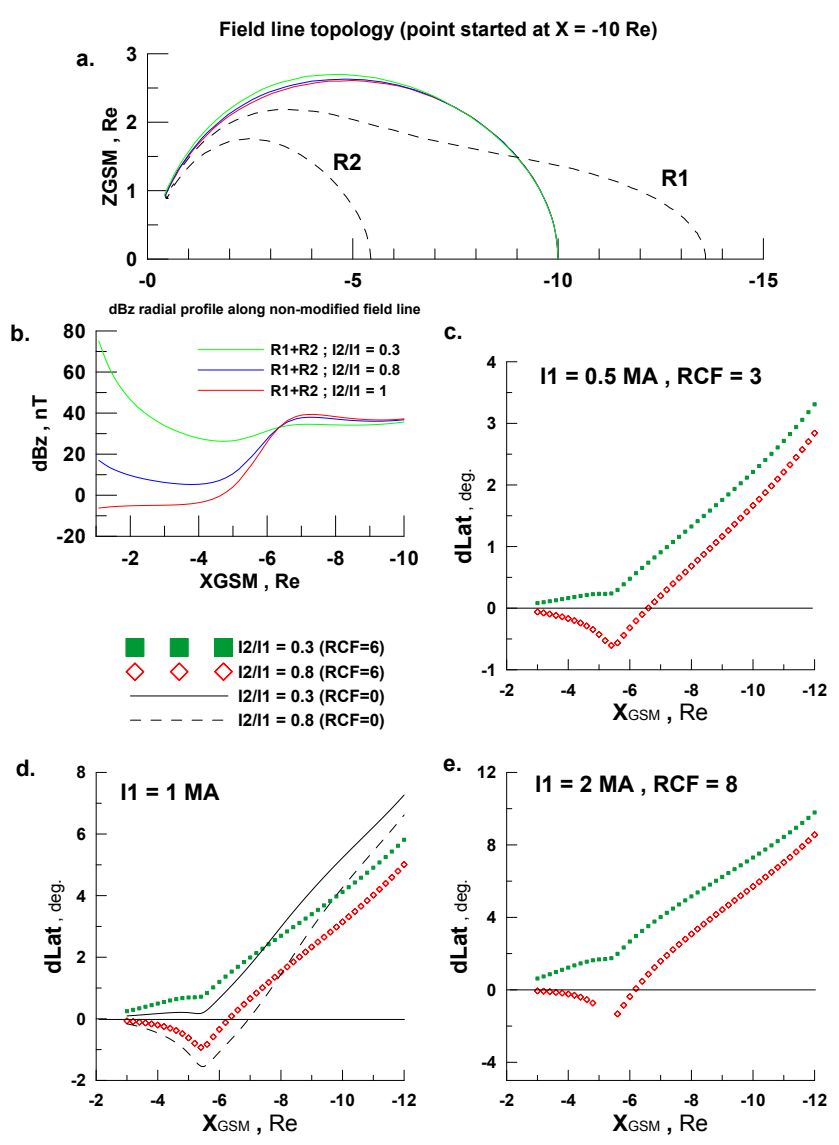

Figure 5. Parameter dependence of footprint displacement. (a) The $X 0 Z$ plane projection of SCW2L FACs (dashed lines) and magnetic field line traced from $X=-10 \mathrm{Re}$; (b) $\Delta B_{Z}$ generated by SCW2L $\left(I_{1}=1 \mathrm{MA}\right)$ along the T89 magnetic field line starting at $X=-10 \mathrm{Re}$; (c) latitudinal shifts for weak substorms $\left(I_{1}=0.5 \mathrm{MA}, \mathrm{RCF}=3\right)$; (d) same panel for strong events with two different magnetotail stretches $\left(I_{1}=1 \mathrm{MA}, \mathrm{RCF}=0\right.$ and 6$)$; (e) same as (c) and (d) but calculated for extremely strong substorms $\left(I_{1}=2 \mathrm{MA}, \mathrm{RCF}=8\right)$. Different colors in panels $(\mathbf{c}-\mathbf{e})$ correspond to the same $I_{1}$ but with two values of the $I_{2} / I_{1}$ current ratio, shown in green for $I_{2} / I_{1}=0.3$ and in red for $I_{2} / I_{1}=0.8$. All calculations are done for the fixed $\mathrm{RT}_{1}=15 \mathrm{Re}, \mathrm{RT}_{2}=6 \mathrm{Re}$, wedge azimuthal width of $50^{\circ}$.

at $X=-11 \operatorname{Re}, Y=3 \operatorname{Re}$, and $Z=-1.56 \operatorname{Re}$ through both events is shown for reference (black lines in Fig. 8a and b). This location (also plotted as a black square in Fig. 7) entered the SCW sector temporarily during both activations. At these times the poleward shifts of this location footpoint (relative to the background location at 04:56 UT) were about 3.5 and $5^{\circ}$, respectively, for the SCW2L model. The maximal poleward shifts near the central meridians of corresponding SCW sectors are about 8 and $11^{\circ}$, according to the diagrams in Fig. 7a and b. Note, that we do not take into account DRP current in our analysis because (1) we actually have no magnetospheric data to evaluate accurately its parameters, (2) its magnitude is three times smaller compared to SCW and (3) we compare observed and predicted expansion by an order of magnitude.

According to the data-based time-varying AM03 model, the maximal poleward shifts were predicted to be smaller, about 1.6 and $4.5^{\circ}$, respectively (see red notches in Fig. $8 \mathrm{~b}$ ). The spatial distribution of the predicted shifts is rather smooth in this case, reflecting the large-scale nature of the model functions in the T96 model. Accordingly, it predicts similar footpoint variations for all GOES spacecraft, irrespective of whether they actually observed the dipolarization. An example is the variation of footpoints of GOES-12 and 14 , which entered the SCW sector and registered the dipolarization at different times.

Another detail to be noted is that the SCW2L and AM03 models predict different footpoint variations during the recovery phase. According to the time-varying adaptive model, the latitude locations of the spacecraft continue to grow when the R1 current starts to drop (regardless of whether the spacecraft stayed inside the dipolarized region). In contrast, the spacecraft footprints calculated using the SCW2L-based model undergo an equatorward shift. Another notable feature is a sharp negative footpoint shift at times when the spacecraft exit from the model SCW (see GOES-14 at around the onset and at $\sim 05: 25 \mathrm{UT}$ ).

Also, even though GOES-12 observed a strong dipolarization $\left(\Delta B_{Z}\right.$ up to $\sim 20 \mathrm{nT}$ ) during the first activation, its footpoint latitude varied only slightly $\left(\sim 0.5^{\circ}\right.$ CGLat $)$ for two reasons. The first is that GOES operates in a region of a strong background magnetic field, which is why the magnitude of spacecraft footprints displacement remains almost unchanged (e.g., Figs. 2 and 7). The situation is different for THEMIS-A, which was located tailward (closer to the R1 current) in a region of a weaker magnetic field (and stronger SCW-related $\Delta B_{Z}$ ) and, correspondingly, in an area of increased mapping distortion. The second is that GOES-12 was located closer to the central SCW meridian, where the effects of FACs are weaker than in regions closer to the edges of the SCW. For this reason, the footprint of GOES-14, which operates in vicinity of upward FACs, has bigger latitudinal variations.

During this substorm, several THEMIS all-sky imagers (ASI) provided useful auroral observations. Although limited by bad weather and moonlight, observations made at postmidnight stations KUUJ and SNKQ distinctly recorded auroral brightening after 04:56 UT (top of Fig. 9). These stations had to be inside the SCW sector according to Fig. 8a. Poleward expansion is clearly limited, and the latitudinal interval of intensified auroras (boundaries of green color in Fig. 9 KUUJ keogram, see white vertical bin) is estimated to be roughly about $\sim 3^{\circ}$ during the first activation. This is comparable to the $\sim 3.5^{\circ}$ poleward expansion predicted by SCW2L the model (see Fig. 8b, vertical bin compared with black line maximum).

At the same time the pre-midnight stations FSMI and SNAP were duskward of the SCW and recorded no active 

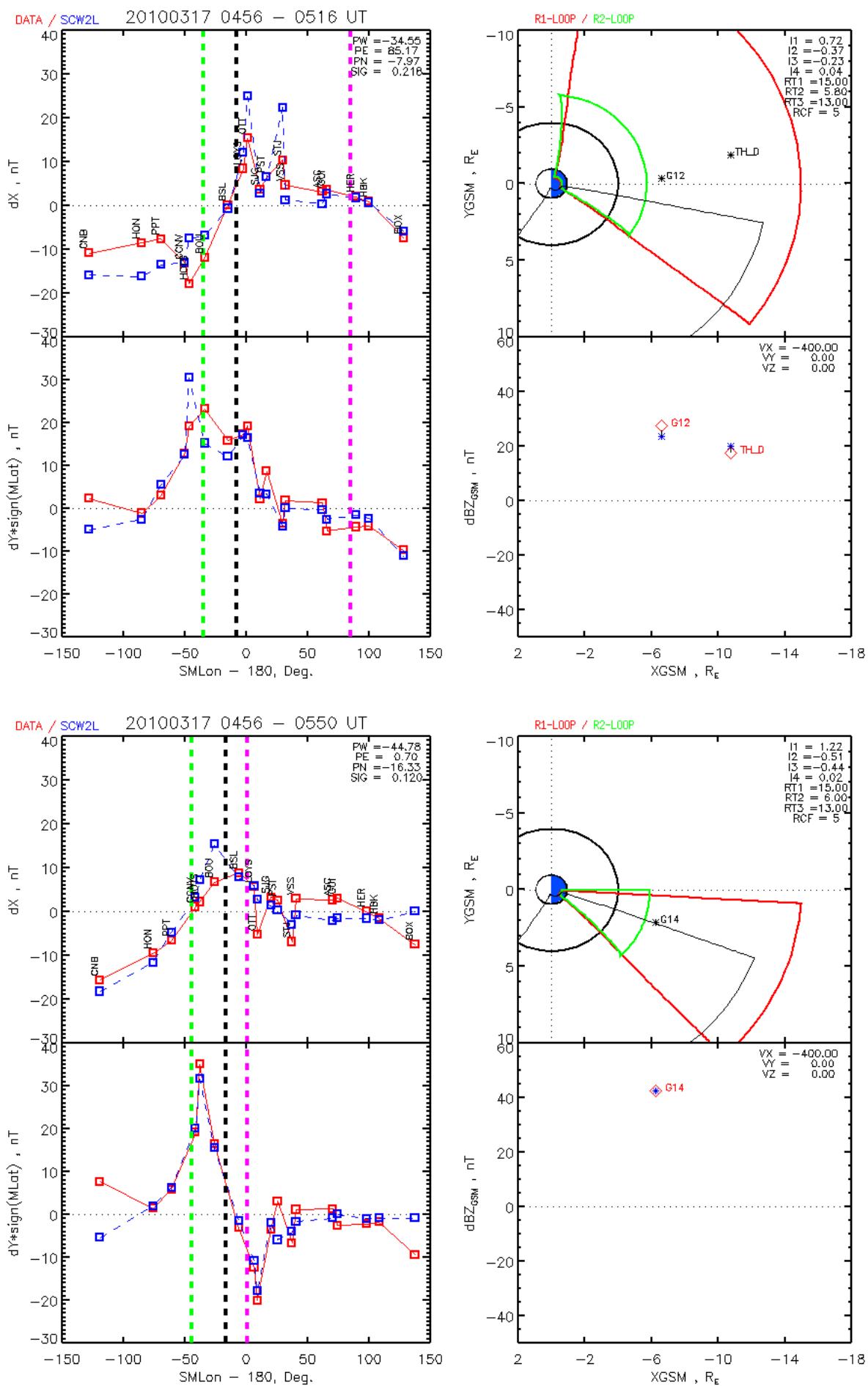

Figure 6. Inversion results for two dipolarization peaks at 05:13 UT (activation no. 1, top) and 05:50 UT (activation no. 2, bottom). The left panels show observed (red) and predicted (blue) ground $\Delta X$ and $\Delta Y$ variation amplitudes; I1, I2, I3, and I4 indicate R1, R2, DRP, and ring current intensities, repsectively. The right bottom panel shows the same for spacecraft $\Delta B_{Z}$ data. The upper right panel illustrates the SCW2L configuration projected onto the $X 0 Y$ plane. 

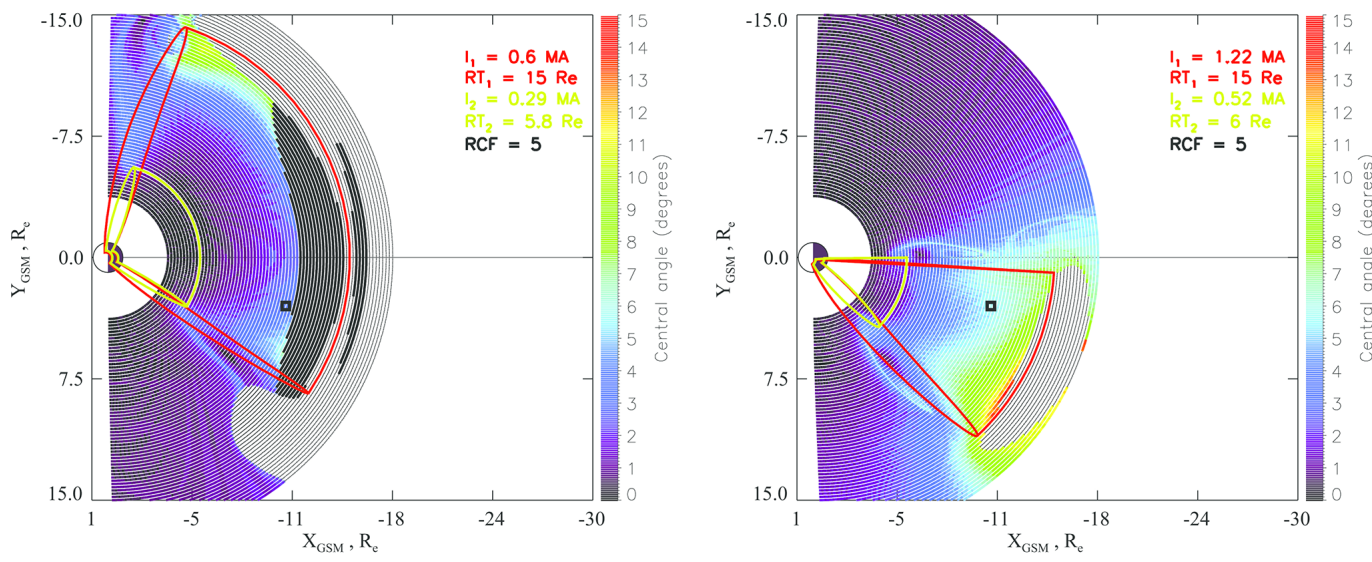

Figure 7. Same as in Fig. 2, but predicted for the peak epochs of two SCW activations, shown in Fig. 6. The black square points indicate dummy spacecraft location relative to the SCW (in the neutral sheet, $X=-11 \operatorname{Re}, \mathrm{Y}=3 \operatorname{Re}$ and $Z=-1.56 \mathrm{Re}$ ).

auroras, but they entered the SCW during second activation according to Fig. 8a. Beginning at 05:36 UT, these stations observed auroral breakup and subsequent extended poleward expansion under good viewing conditions. The breakup started $\sim 50 \mathrm{~km}$ south of FSMI station zenith at $67.4^{\circ}$ CGLat. It is also seen at the equatorward horizon, $\sim 4^{\circ}$ CGLat south of SNAP station located at $71.0^{\circ}$ CGLat at the same meridian. The bright auroras expanded poleward to the northern horizon, suggesting a roughly $\sim 8^{\circ}$ CGLat poleward shift during the second activation. This number is slightly larger than in our SCW2L predictions, which give $\sim 5^{\circ}$ CGLat. The AM03 model provides an even smaller value of $\sim 4.5^{\circ}$. Our modeling indicates that the deformation of magnetic configuration by SCW currents provides more than half of the observed poleward expansion. The remaining part can be ascribed to tailward motion of the magnetic reconnection region (and of the current disruption region), that is, to an effect that could not be taken into account in our data-based modeling.

\section{Discussion and concluding remarks}

A few previous studies concluded that field-aligned currents, having realistic strength and distribution, may considerably affect ionospheric mapping of equatorial magnetospheric points and ionospheric images of magnetospheric structures (e.g., Vasilyev et al., 1986; Kaufmann and Larson, 1989; Donovan, 1993; Tsyganenko, 1997). These examples utilized simplified models of filamentary and/or distributed currents. Kaufmann and Larson (1989) constructed FAC models as a combination of a number of current wires and used this model to map magnetic field lines, electric fields, and equipotentials throughout the magnetosphere. Near intense region 1 and region 2 Birkeland currents, they found large magnetic footpoint displacements and discussed the importance of twisting the magnetic field lines to form spiral patterns in the regions co-located with the WTS and at the eastward end of the substorm current system. By modeling finite thickness field-aligned current sheets connected via radial or azimuthal currents in the magnetosphere, Donovan (1993) emphasized the large amplitude of footpoint distortions and the crucial dependence of distortion type and mapping on the character of FAC closure in the magnetosphere (of which very little is known). Tsyganenko (1997) developed a mathematical approach to construct electric current flow lines, the prototype of which was based on two inclined, tailward-shifted circular loops. Using this model, the author mapped a set of equatorial circular contours to the ionosphere, equidistantly distributed in the equatorial plane between 5 and $20 \mathrm{Re}$. A conspicuous bulge-like form was shown to emerge in the nightside ionosphere inside the SCW sector, where the magnetic field lines collapsed towards a more dipole-like configuration.

Specific magnetic field line distortions resulting from the growth an SCW-like current system were addressed by Vasilyev et al. (1986), who calculated the current system's magnetic effects using a wire-type SCW model with currents flowing along stretched field lines described by the empirical T87 magnetospheric model (Tsyganenko, 1987). Their results are most relevant to the results of our study. In particular these authors mapped neutral sheet locations to the ionosphere, identified mapping patterns that are similar to the auroral bulge (like those shown in our Fig. 3), and found that the magnitude of footprint poleward shift due to the R1 type current of $I_{1}=1 \mathrm{MA}$ may reach $7^{\circ}$ CGLat. According to their results, FAC-related magnetic field distortions are strong enough to potentially explain poleward auroral expansion and shape of the auroral bulge, including the WTS formation. Our investigation includes a more accurate finitesize filamentary model for the field-aligned currents, that is, a better (more accurate) empirical model to describe both the background and the FAC field-line skeleton (see Paper 1), and more sophisticated and realistic (two-loop) construction of the SCW model. With its model flexibility and spacecraft 

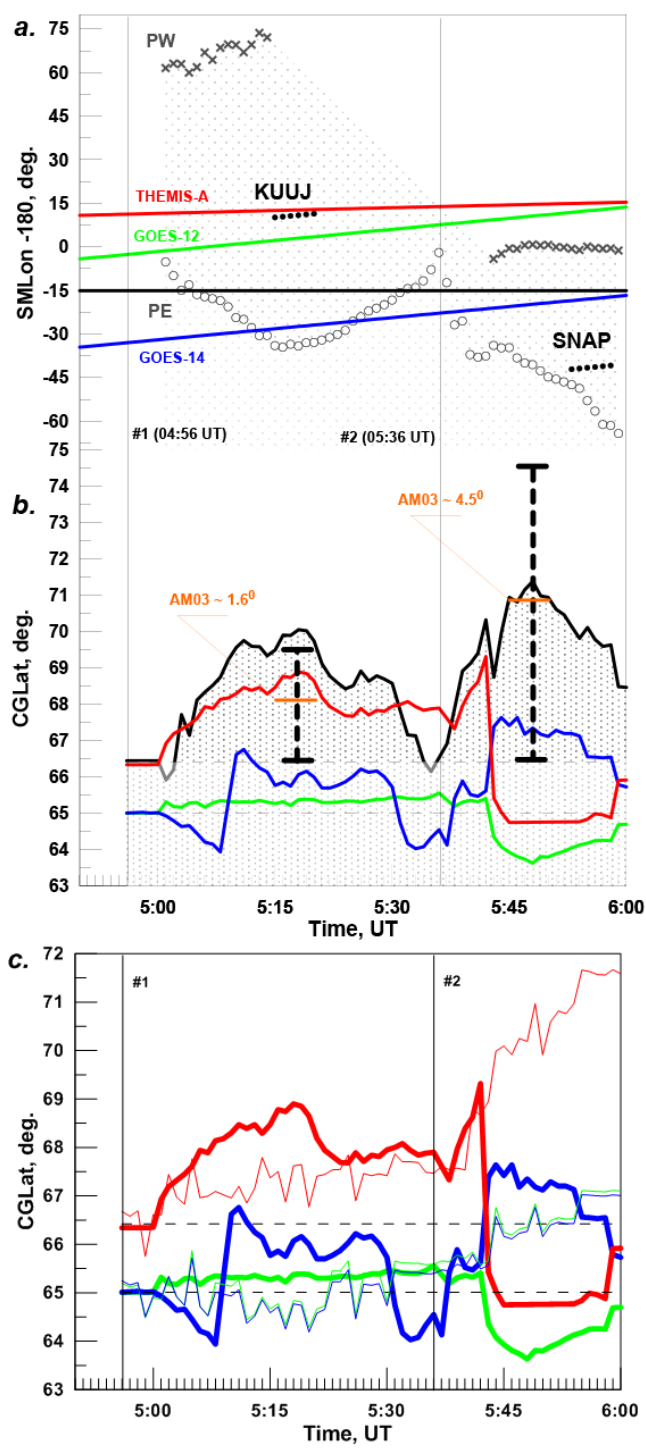

Figure 8. (a) THEMIS and GOES spacecraft, KUUJ and SNAP station locations relative to SCW; (b) CGLat variations of spacecraft ionospheric footpoints caused by SCW2L (colored solid lines) during activations no. 1 and no. 2; (c) comparison of CGLat footprints variations predicted by the SCW2L (thick lines) and time-varying adaptive AM03 models (thin lines). The black line in panel (b) indicates the ionospheric position of dummy spacecraft at $X=-11 \mathrm{Re}$, $Y=3 \mathrm{Re}$, and $Z=-1.56 \mathrm{Re}$ (neutral sheet, see also black square in Fig. 7a and b). Vertical bars illustrate the amplitude of the auroral poleward expansion observed by FSMI and SNAP ground magnetometers. The ginger notches show AM03 predictions (at dipolarization peaks) for the dummy spacecraft located at $R=11 \mathrm{Re}$. The footprint calculation time covers both dipolarizations from $T=04: 30$ to $05: 58 \mathrm{UT}$.

data coverage, our approach has a greater chance of validating the model. Because of these improvements, we can confirm the main findings of Vasilyev et al. (1986) and particularly confirm that the intensity of R 1 current plays the main

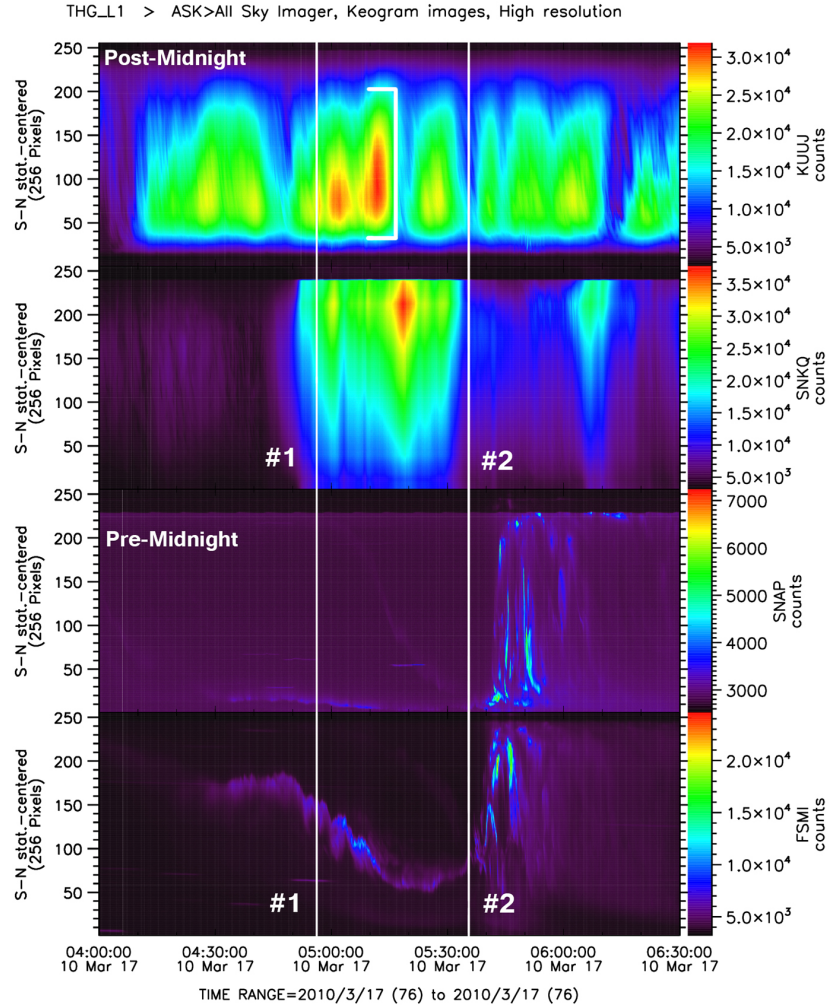

Figure 9. THEMIS ground-based all sky imager (ASI) observations. From top to bottom: Keograms of post-midnight stations KUUJ and SNKQ and pre-midnight stations SNAP and FSMI.

role in magnetospheric magnetic field and mapping distortions.

The mapping problem was addressed differently by Kubyshkina et al. (2011). As in our effort described in Sect. 3, they fit the time-varying AM03 model to observations during a substorm made with good spacecraft coverage. Based on that model, they found that locations of the spacecraft footprints undergo changes from magnetic field distortion and are similar to variations in the poleward edge of bright auroras. However, as with our case, the AM03based technique, which uses large-scale current distributions, is not capable of reproducing localized dipolarization and the azimuthally confined auroral-bulge shape of footpoint distortions. The Kubyshkina et al. (2011) result also crucially depends on spacecraft coverage and details of the spacecraft location with respect to the location and evolution of magnetospheric activity. The hypothesis that magnetic field distortions are capable of producing significant bulge-like mapping displacement is also consistent with first-principlebased simulations of fast-flow intrusion into the inner magnetosphere (Yang et al. 2012, Birn and Hesse 2013). These simulations also confirm the quadrupolar (combination of R1 and R2 like loops) field-aligned current distributions associated with localized dipolarizations. 
Our investigation explored the effects of the substorm current wedge parameters on the geometry of ionospheric mapping from the magnetospheric equatorial plane using the advanced substorm current wedge model (SCW2L). It provides a few conclusions important for understanding mapping distortions and their implications for dynamics and structure of bright auroras during the substorms.

1. The mapping from the dipolarized region provides evidence for the poleward shift of ionospheric footpoints (see Figs. 2, 3 and 5). The magnitude of the latitudinal shift depends primarily on the R1 loop current intensity $\left(I_{1}\right)$, which controls the dipolarization magnitude in the magnetosphere. The footpoint displacement, which may reach $10^{\circ} \mathrm{CGLat}$ during extremely strong substorms $\left(I_{1}=2 \mathrm{MA}\right)$ and is minimized $\left(<3^{\circ}\right)$ during weak disturbances $\left(I_{1}<0.5 \mathrm{MA}\right)$, gives a realistic range of auroral expansion sizes during substorms (Akasofu, 1976; Yahnin, 2006). Modeling of a substorm event on 17 March 2010 confirms that poleward shift values ( $\sim 3.5$ and $\sim 5^{\circ}$ CGLat) predicted from databased SCW2L modeling results are comparable to (or are somewhat less than) the poleward auroral expansion observed by the ground ASI network during two consecutive activations ( $\sim 3$ and $\sim 7 \ldots 8^{\circ}$ CGLat). The difference is ascribed to tailward propagation of the reconnection/disruption region during the final substorm stage (e.g., Baumjohann et al., 1999).

2. The helical magnetic field twists magnetic field lines near FAC filaments and forms medium-size surges at the dusk and dawn side boundaries of the current wedge (of the azimuthally localized dipolarization region). The surge size is comparable to the magnitude of the abovementioned poleward expansion (Fig. 3). The surge around intense upward FACs, where electrons are expected to be accelerated into the ionosphere by the intense field-aligned electric field, explains the bright westward travelling surge, a remarkable substorm-related mesoscale auroral structure. It should also be noted that we do not consider the spiral structure at the eastward bulge termination, because in reality it corresponds to diffuse auroras (region of active proton precipitation) and downward FACs, which are azimuthally wider than upward FACs and cannot be represented by a current filament. The spiral form of the aurora is usually observed near the upward FACs, but rarely near the downward FACs.

3. Images of straight-shaped magnetospheric flow channels or structures can be significantly distorted (twisted, rotated) near intense field-aligned currents with largescale geometry (see also Vasilyev et al., 1986; Kaufmann and Larson, 1989; Donovan, 1993). The effect strongly depends on the relative location of the structures with respect to the filamentary FACs. Magnetic field twisting effect may partly cause azimuthal deflection of auroral streamers approaching diffuse auroras (Nakamura et al., 1993; Nishimura et al., 2010), although true flow deflection in the azimuthal direction may also contribute to this effect (Lyons et al., 2012). An illustration of the spiral structure around upward FACs was provided by FREJA satellite measurements of multiple spiral arcs and the associated rotating electric fields in the WTS region were published in Marklund et al. (1998).

4. In the near-Earth part of the azimuthal sector occupied by the SCW earthward of the inner edge of the dipolarized region (where particle injection also takes place), footpoint poleward expansion is largely suppressed by the counter-effect of the R2 current loop. In cases with strong R2 current (e.g., with $I_{2} / I_{1}=0.8$ in Fig. 4, or larger), the R2 loop field-aligned current may even produce the equatorward shift and rotation of ionospheric footpoints, causing a small equatorward footpoint bulge (see, e.g., Fig. 3). It is not clear the R2 current may be this strong; more study is required. If this effect exists, it may contribute to the modest $\left(\sim 1-2^{\circ}\right.$ CGLat) equatorward expansion of diffuse structured auroras that has been observed (e.g., Nakamura et al., 1993; Keiling et al., 2012), but has not been extensively studied.

\section{The Supplement related to this article is available online at doi:10.5194/angeo-33-505-2015-supplement.}

Acknowledgements. This study was supported by the EU FP7 grant 263325 (ECLAT) and RFBR grant no. 14-05-31472. We thank J. Hohl (Department of Earth, Planetary, and Space Sciences, UCLA) for help with editing of the manuscript. We also thank INTERMAGNET project (http://intermagnet.org) for providing ground magnetometer data and CDAWeb (http://cdaweb.gsfc. nasa.gov) data base for providing spacecraft and auroral observations.

The topical editor E. Roussos thanks the two anonymous referees for help in evaluating this paper.

\section{References}

Akasofu, S.: Recent progress in studies of DMSP auroral photographs, Space Sci. Rev., 19, 169-215, 1976.

Angelopoulos, V.: The THEMIS mission, Space Sci. Rev., 141, 534, doi:10.1007/s11214-008-9336-1, 2008.

Angelopoulos, V., Kennel, C. F., Coroniti, F. V., Pellat, R., Kivelson, M. G., Walker, R. J., Baumjohann, W., Paschmann, G., and Luhr, H.: Bursty bulk flows in the inner plasmasheet: An effective means of earthward transport in the magnetotail, in: Proceedings of the First International Conference on Substorms (ICS-1), Kiruna, Sweden, ESA Spec. Publ., ESA SP-335, 303, 1992. 
Baumjohann, W., Paschmann, G., and Lühr, H.: Characteristics of high-speed ion flows in the plasma sheet, J. Geophys. Res., 95, 3801-3809, doi:10.1029/JA095iA04p03801, 1990.

Baumjohann, W., Hesse, M., Kokubun, S., Mukai, T., Nagai, T., and Petrukovich, A.: Substorm dipolarization and recovery, J. Geophys. Res., 104, 24995-25000, 1999.

Birn, J. and Hesse, M.: The substorm current wedge in MHD simulations, J. Geophys. Res.-Space, 118, 3364-3376, doi:10.1002/jgra.50187, 2013.

Birn, J. and Hesse, M.: The substorm current wedge: Further insights from MHD simulations, J. Geophys. Res., 119, 35033513, doi:10.1002/2014JA019863, 2014.

Birn, J., M. Hesse, G. Haerendel, W. Baumjohann, and K. Shiokawa, Flow braking and the substorm current wedge, J. Geophys. Res., 104, 19895-19903, 1999.

Chu, X., Hsu, T.-S., McPherron, R. L., Angelopoulos, V., Pu, Z., Weygand, J. J., Khurana, K., Connors, M., Kissinger, J., Zhang, H., and Amm, O.: Development and validation of inversion technique for substorm current wedge using ground magnetic field data, J. Geophys. Res.-Space, 119, 1909-1924, doi:10.1002/2013JA019185, 2014.

Donovan, E. F.: Modeling the magnetic effects of fieldaligned currents, J. Geophys. Res., 98, 13529-13543, doi:10.1029/93JA00603, 1993.

Elphinstone, R. D., Murphree, J. S., and Cogger, L. L.: What is a global auroral substorm?, Rev. Geophys., 34, 169-232, doi:10.1029/96RG00483, 1996.

Henderson, M. G.: Auroral Substorms, Poleward Boundary Activations, Auroral Streamers, Omega Bands, and Onset Precursor Activity, in: Auroral Phenomenology and Magnetospheric Processes: Earth And Other Planets, edited by: Keiling, A., Donovan, E., Bagenal, F., and Karlsson, T., American Geophysical Union, Washington, D.C., USA, 39-54, doi:10.1029/2011GM001165, 2012.

Henderson, M. G., Reeves, G. D., and Murphee, J. S.: Are northsouth aligned auroral structures an ionospheric manifestation of bursty bulk flows?, Geophys. Res. Lett., 25, 3737-3740, 1998.

Horning, B., McPherron, R., and Jackson, D.: Application of linear inverse theory to a line current model of substorm current systems, J. Geophys. Res., 79, 5202-5210, 1974.

Iijima, T. and Potemra, T. A.: The amplitude distribution of fieldaligned currents at northern high latitudes observed by triad, J. Geophys. Res., 81, 2165-2174, doi:10.1029/JA081i013p02165, 1976.

Kaufmann, R. L. and Larson, D. J.: Electric field mapping and auroral Birkeland currents, J. Geophys. Res., 9, 15307, doi:10.1029/JA094iA11p15307, 1989.

Keiling, A., Shiokawa, K., Uritsky, V., Sergeev, V., Zesta, E., Kepko, L., and Østgaard, N.: Auroral Signatures of the Dynamic Plasma Sheet, in: Auroral Phenomenology and Magnetospheric Processes: Earth And Other Planets, edited by: Keiling, A., Donovan, E., Bagenal, F., and Karlsson, T., American Geophysical Union, Washington, D.C., USA, 317-335, doi:10.1029/2012GM001231, 2012.

Kubyshkina, M. V., Sergeev, V. S., Tsyganenko, N. A., Angelopoulos, V., Runov, A., Donovan, E., Singer, H., Auster, U., and Baumjohann, W.: Time-dependent magnetospheric configuration and breakup mapping during a substorm, J. Geophys. Res., 116, A00I27, doi:10.1029/2010JA015882, 2011.
Liu, J., Angelopoulos, V., Runov, A., and Zhou, X.-Z.: On the current sheets surrounding dipolarizing flux bundles in the magnetotail: The case for wedgelets, J. Geophys. Res.-Space, 118, 20002020, doi:10.1002/jgra.50092, 2013.

Lyons, L. R., Nagai, T., Blanchard, G. T., Samson, J. C., Yamamoto, T., Mukai, T., Nishida, A., and Kokubun, S.: Association between Geotail plasma flows and auroral poleward boundary intensifications observed by CANOPUS photometers, J. Geophys. Res., 104, 4485-4500, doi:10.1029/1998JA900140, 1999.

Lyons, L. R., Nishimura, Y., Xing, X., Shi, Y., Gkioulidou, M., Wang, C.-P., Kim, H.-J., Zou, S., Angelopoulos, V., and Donovan, E.: Auroral Disturbances as a Manifestation of Interplay Between Large-Scale and Mesoscale Structure of Magnetosphere-Ionosphere Electrodynamical Coupling, in: Auroral Phenomenology and Magnetospheric Processes: Earth And Other Planets, edited by: Keiling, A., Donovan, E., Bagenal, F., and Karlsson, T., American Geophysical Union, Washington, D.C., USA, 193-204, doi:10.1029/2011GM001152, 2012.

Marklund, G. T., Karlsson, T., Blomberg, L. G., Lindqvist, P.-A., Falthammar, C.-G., Johnson, M. L., Murphree, J. S., Andersson, L., Eliasson, L., Opgenoorth, H. J., and Zanetti, L. J.: Observations of the electric field fine structure associated with the westward traveling surge and large-scale auroral spirals, J. Geophys. Res., 103, 4125-4144, doi:10.1029/97JA00558, 1998.

McPherron, R. L., Russell, C. T., and Aubry, M. A.: Phenomenological model for substorms, J. Geophys. Res., 78, 3131-3149, doi:10.1029/JA078i016p03131, 1973.

Nakamura, R., Oguti, T., Yamamoto, T., and Kokubun, S.: Equatorward and poleward expansion of the auroras during auroral substorms, J. Geophys. Res., 98, 5743-5759, 1993.

Nakamura, R., Baumjohann, W., Brittnacher, M., Sergeev, V. A., Kubyshkina, M. V., Mukai, T., Liou, K.: Flow bursts and auroral activations: onset timing and foot point location, J. Geophys. Res., 106, 10777-10789, doi:10.1029/2000JA000249, 2001.

Nishimura, Y., Lyons, L., Zou, S., Angelopoulos, V., and Mende, S.: Substorm triggering by new plasma intrusion: THEMIS all-sky imager observations, J. Geophys. Res., 115, A07222, doi:10.1029/2009JA015166, 2010.

Roux, A., Perraut, S., Robert, P., Morane, A., Pedersen, A., Korth, A., Kremser, G., Aparicio, B., Rodgers, D., and Pellinen, R.: Plasma sheet instability related to the westward traveling surge, J. Geophys. Res., 96, 17697-17714, doi:10.1029/91JA01106, 1991.

Sergeev, V. A., Vagina, L. I., Elphinstone, R. D., Murphee, J. S., Hearn, D. J., and Johnson, M. L.: Comparison of UV optical signatures with the substorm current wedge predicted by an inversion algorithm, J. Geophys. Res., 101, 2615-2627, 1996.

Sergeev, V. A., Tsyganenko, N. A., Smirnov, M. V., Nikolaev, A. V., Singer, H. J., and Baumjohann, W.: Magnetic effects of the substorm current wedge in a "spread-out wire" model and their comparison with ground, geosynchronous, and tail lobe data, J. Geophys. Res., 116, A07218, doi:10.1029/2011JA016471, 2011.

Sergeev, V. A., Nikolaev, A. V., Tsyganenko, N. A., Angelopoulos, V., Runov, A. V., Singer, H. J., and Yang, J.: Testing a two-loop pattern of the substorm current wedge (SCW2L), J. Geophys. Res.-Space, 119, doi:10.1002/2013JA019629, 2014a.

Sergeev, V. A., Nikolaev, A. V., Kubyshkina, M.V., Tsyganenko, N.A., Singer, H., Rodriguez, J., Angelopoulos, V., Nakamura, R., Milan, S., Coxon, J., Anderson, B., and Korth, H.: Event 
study combining magnetospheric and ionospheric perspectives of the substorm current wedge modeling and dynamics, J. Geophys. Res.-Space, 119, 9714-9728, doi:10.1002/2014JA020522, 2014b.

Tsyganenko, N. A.: Global quantitative models of the geomagnetic field in the cislunar magnetosphere for different disturbance levels, Planet. Space Sci., 35, 1347-1358, 1987.

Tsyganenko, N. A.: A magnetospheric magnetic field model with warped tail current sheet, Planet. Space Sci., 37, 5-20, 1989.

Tsyganenko, N. A.: An empirical model of the substorm current wedge, J. Geophys. Res., 102, 19935-19941, 1997.

Vasilyev, E. P., Sergeev, V. A., and Malkov, M. V.: Threedimensional effects of the Birkeland current loop, Geomagn. Aeron., 26, 114-118, 1986.
Waters, C. L., Anderson, B. J., and Liou, K.: Estimation of global field aligned currents using the Iridium system magnetometer data, Geophys. Res. Lett., 28, 2165-2168, doi:10.1029/2000GL012725, 2001.

Yahnin, A. G., Despirak, I. V., Lubchich, A. A., Kozelov, B. V., Dmitrieva, N. P., Shukhtina, M. A., and Biernat, H. K., Relationship between substorm auroras and processes in the near-Earth magnetotail, Space Sci. Rev., 122, 97-106, doi:10.1007/s11214006-5884-4, 2006.

Yang, J., Toffoletto, F. R., Wolf, R. A., Sazykin, S., Ontiveros, P. A., and Weygand, J. M.: Large-scale current systems and ground magnetic disturbance during deep substorm injections, J. Geophys. Res., 117, A04223, doi:10.1029/2011JA017415, 2012. 